\title{
Group B streptococcus colonisation in pregnant women at Dr. George Mukhari Hospital, South Africa
}

\author{
MC Monyama ${ }^{a}$, JY Bolukaoto ${ }^{a}$, MO Chukwu ${ }^{a}$, MRB Malobab, SR Moyoc, RT Mavenyengwa ${ }^{c, d^{*}}$, M Nchabeleng ${ }^{b}$ and SL Lebeloa \\ ${ }^{a}$ Department of Life and Consumer Sciences, University of South Africa (UNISA), Florida, South Africa \\ ${ }^{b}$ Department of Microbiological Pathology, Sefako Makgatho Health Sciences University, Pretoria, South Africa \\ 'Department of Health Sciences, Faculty of Health and Applied Sciences, Namibia University of Science and Technology, Windhoek, Namibia \\ ${ }^{d}$ Department of Medical Microbiology, University of Zimbabwe College of Health Sciences, Harare, Zimbabwe \\ *Corresponding author, email: rmavenyengwa@yahoo.com
}

The aim of the study was to estimate group B streptococcus (GBS) colonisation in pregnant mothers using selective enrichment broth and solid media for culturing GBS. Vaginal and rectal swabs were collected from 413 pregnant women for GBS culture at recruitment stage. Direct plating and enrichment broth culture methods were compared by using the same swab samples. The swabs were cultured on colistin nalidixic agar (CNA) plate and incubated at $37^{\circ} \mathrm{C}$ and examined after $18-24 \mathrm{~h}$. The samples which were culture negative on a CNA agar plate were then inoculated into a Todd-Hewitt enrichment broth to recover any GBS present that was not recovered on the solid agar. With the CNA agar plate, the samples were cultured separately to enable identification of colonised sites such as vaginal sites or rectal sites. Rectal and vaginal swabs were inoculated into Todd-Hewitt enrichment broth at the same time in the same tube. The GBS colonisation rate in pregnant women was 30.9\% (128/413). The CNA agar plate recovered $45.3 \%(58 / 128)$ of the GBS isolates, whereas $54.7 \%(70 / 128)$ isolates were recovered from Todd-Hewitt broth. Pregnant women of various ages were found to be at risk of GBS colonisation. The colonisation rate was however highest among women of 25-29 age groups as compared with other age groups. Detection of group B streptococcus improved when both rectal and vaginal swabs were collected for laboratory analysis. The simultaneous use of Todd-Hewitt broth and CNA plate also improved the yield of group B streptococcus.

Keywords: colonisation, detection methods, Group B streptococcus, pregnant women

\begin{abstract}
Introduction
Streptococcus agalactiae (Group B streptococcus; GBS) is an encapsulated organism capable of infecting newborn babies, and can produce severe disease in immunocompromised hosts. ${ }^{1}$ Infection is rare in immunocompetent patients except among those with underlying conditions. ${ }^{2}$ The major colonised sites for GBS are reported to be the vagina and the rectum. The colonisation of these regions is a risk factor for subsequent infection in pregnant women and newborns., ${ }^{3,4}$ Ethnicity, maternal age, parity, marital status, education and smoking have been reported not to influence the prevalence of colonisation of GBS. ${ }^{5}$
\end{abstract}

The study done by Dangor et al. confirmed the high incidence of invasive GBS disease observed in the last two decades in parts of Africa. ${ }^{6}$ Maternal screening for GBS recto-vaginal colonisation during pregnancy in South Africa is still not routinely performed in public health facilities. ${ }^{7}$ The high incidence might be attributed to the prevalence of HIV as an underlying condition predisposing patients to GBS infection.

The screening-based strategy can reduce early onset of neonatal GBS disease. The strategy's effectiveness depends on the specimen sampling site, the timing of collection and sensitivity of the microbiological methods used. ${ }^{8}$ The centre for Disease Control and Prevention (CDC) recommends that isolation of group B streptococcus from vaginal and rectal swabs should be done in a selective broth medium (Todd-Hewitt broth with gentamicin, colistin and nalidixic acid) followed by subculture on blood agar or selective media. ${ }^{9}$ Sensitivity of selective broth medium for GBS was reported to be 82 to $99 \% .{ }^{8,10,11}$ Different strategies for identification of high risk mothers and infants and provision of intrapartum prophylaxis may reduce the rate of neonatal sepsis, though they are unlikely to eliminate the problem. ${ }^{12}$ Limitations to the use of intrapartum antibiotics include the emergence of antibiotic-resistant GBS and ineffectiveness in preventing late onset disease due to absence of protective antibodies in mothers. ${ }^{13}$

The aim of this study was to assess the colonisation rate of GBS and the associated risk factors in pregnant women at Dr. George Mukhari Academic Hospital.

\section{Method \\ Study area}

The study was conducted at an antenatal clinic at Dr. George Mukhari Academic Hospital in Ga-Rankuwa, Pretoria, which services the city of Tshwane municipality in Gauteng, South Africa. The hospital is a teaching hospital for the Sefako Makgatho Health Sciences University (SMU), formerly known as the University of Limpopo (Medunsa campus). Ga-Rankuwa has a mixed population comprising various black African tribal groups. The hospital's antenatal clinic and paediatric outpatient department serve the immediate surrounding areas and distant areas such as Limpopo and North West provinces.

\section{Study design and population}

This was a descriptive, prospective study, and the participants were pregnant women who were able to provide informed consent for participation. The women were at least 18 years of age and were assessed to be at 16 weeks gestational age and above. Women who had received antibiotic treatment in the 
2 weeks prior to recruitment, and who were regarded as critically ill and admitted by the attending physician, were excluded from the study. A convenient sampling method was used. Vaginal (lower and higher vaginal) and rectal swabs were collected from 413 pregnant women between February 2012 and October 2012 by a trained research nurse. Group B streptococcus colonisation was defined as isolation of GBS from at least one type of sample.

Data on socio-economic status (level of education, employment, and marital status), previous and current obstetric history (parity, miscarriages, stillbirths) and current health status were collected using a questionnaire at recruitment.

\section{Sample collection and processing}

Specimens were collected using a cotton wool swab and placed in Amies transport medium without charcoal (Rochelle Chemicals \& Lab equipment, South Africa). All samples were accurately labelled and placed in a cooler box filled with ice blocks and transported from the antenatal clinic to Dr. George Mukhari Academic Hospital National Health Laboratory Science (NHLS) Medical Microbiology laboratory for processing within $2 \mathrm{~h}$.

All vaginal and rectal swab specimens were cultured on colistin nalidixic agar (CNA) plates, and into Todd-Hewitt broth supplemented with $8 \mu \mathrm{g} / \mathrm{ml}$ colistin and $15 \mu \mathrm{g} / \mathrm{ml}$ gentamicin to inhibit other normal flora and recover GBS that may not have been detected on CNA. ${ }^{14}$ Low vaginal swabs were those that were inserted into the vagina with a depth less than $2 \mathrm{~cm}$, whereas high vaginal swabs were those that were inserted with a depth greater than $2 \mathrm{~cm}$. The plates and tubes were incubated overnight at $37^{\circ} \mathrm{C}$ in the presence of $5 \%$ carbon dioxide. After 18 24 hours of incubation, primary plates were examined for $\beta$ haemolytic streptococcal colonies typical of GBS. A single colony from each isolate was picked and subcultured onto blood agar. Plates showing no growth were incubated for another $24 \mathrm{~h}$. Todd Hewitt broth cultures were inoculated onto $5 \%$ sheep blood agar plates after overnight incubation. Two CNA agar plates were used to culture the swabs, one plate was divided into two to culture high vaginal and lower vaginal swabs and another plate was used for rectal swab culture. Isolates were identified as GBS by the following criteria: $\beta$-haemolysis on blood agar, Gram staining showing Gram-positive cocci in pairs or short chains, negative reaction with catalase reagent, bile esculin agar and Lancefield grouping with group B antisera (Streptex; Biomereux and Remel Ltd, France). If the results were not clear, CAMP (Christie Atkins Munch-Petersen) test was employed to confirm group B streptococcus isolates. Data analysis of sociodemographic factors was done using the Chi-squared test at a significance level of $p<0.05$.

\section{Ethical approval}

Informed consent was obtained from the patients and the human experimentation guidelines of the South African Medical Research Council (MRC) were followed in the conduct of the research. The study was approved by Higher Degrees Ethics Committee and SMU/Medunsa Research and Ethics Committee, Dr. George Mukhari Academic Hospital Management (MREC/P/02/2011: IR) and UNISA College of Agriculture and Environmental Sciences.

\section{Results}

GBS colonisation was detected in $30.9 \%$ (128/413) of the pregnant women. All the women enrolled in the study were of the same racial group (black Africans) with an age range of 18-45 years and mean age of 30 . The study participants were from different geographic areas (urban, semi-urban and rural) surrounding Dr. George Mukhari Academic Hospital. The majority of the participants were single, widowed or divorced. The highest proportion of GBS colonised women was among those of the age group between 25 and 29 (Table 1). The $p$-values were computed after comparative analysis of the risk factors for each socio-demographic characteristic.

Most participants had an educational level below matric (56.8\%), with the fewest participants having a tertiary level education (16.6\%). The difference in colonisation rates were significant

Table 1: Socio-demographic characteristics of pregnant women investigated for GBS colonisation

\begin{tabular}{|c|c|c|c|c|}
\hline \multirow[t]{2}{*}{ Characteristics } & \multirow[t]{2}{*}{ Category } & GBS colonised $(n=128)$ & GBS non-colonised $(n=285)$ & \multirow[t]{2}{*}{$\boldsymbol{p}$-value } \\
\hline & & Frequency (\%) & Frequency (\%) & \\
\hline \multirow[t]{6}{*}{ Age in years } & $<20$ & $2(1.6)$ & $10(3.5)$ & \multirow[t]{6}{*}{0.07} \\
\hline & $20-24$ & $28(21.8)$ & $47(16.5)$ & \\
\hline & $25-29$ & $39(30.5)$ & $71(24.9)$ & \\
\hline & $30-34$ & $24(18.8)$ & $95(33.3)$ & \\
\hline & $35-39$ & $24(18.8)$ & $57(20.0)$ & \\
\hline & $\geq 40$ & $10(7.8)$ & $20(7.0)$ & \\
\hline \multirow[t]{3}{*}{ Geographical areas } & Urban & $113(88.8)$ & $246(86.3)$ & \multirow[t]{3}{*}{0.08} \\
\hline & Urban-rural & $8(1.9)$ & $28(9.8)$ & \\
\hline & Rural & $4(1.0)$ & $2(0.7)$ & \\
\hline \multirow[t]{3}{*}{ Marital status } & Married & $27(21.1)$ & $71(17.2)$ & \multirow[t]{3}{*}{0.56} \\
\hline & Cohabiting & $31(24.2)$ & $58(14.0)$ & \\
\hline & Single/widowed/divorced & $67(52.3)$ & $147(35.6)$ & \\
\hline \multirow[t]{3}{*}{ Educational level } & Below matric & $71(55.5)$ & $107(37.5)$ & \multirow[t]{3}{*}{$<0.0001$} \\
\hline & Matric & $35(27.3)$ & 153(53.7) & \\
\hline & Tertiary & $15(11.7)$ & $15(5.2)$ & \\
\hline \multirow[t]{2}{*}{ Employment } & Unemployed & $46(35.9)$ & $179(62.8)$ & \multirow[t]{2}{*}{0.003} \\
\hline & Employed & $51(39.8)$ & $97(34.0)$ & \\
\hline
\end{tabular}


among the women at different educational levels $(p<0.05)$. There was also a significant difference in colonisation rates between unemployed women compared with employed ones $(p<0.05)$.

The age range of the GBS colonised pregnant women was 18-45 and the age categories were compared. The GBS colonisation rate in pregnant women was highest in the 25-29 year age group, and lowest in the $<20$ year age category. Adverse pregnancy outcomes were categorised into miscarriages, stillbirths and HIVpositive as an underlying condition. Pregnant women who were currently colonised with GBS and had miscarriages previously comprised $25.0 \%(32 / 128)$ while $9.4 \%(12 / 128)$ had stillbirths (Table 2). N/A means the number of women who were not categorised based on trimester as this data was not available. The $p$-values were computed after comparative analysis of the risk factors for each variable. None of the risk factors were significantly associated with GBS colonisation.

\section{Comparison between media and site used for diagnosis}

The importance of selective enrichment broth and selective plating is shown in Table 3. Slightly more than half of GBSpositive samples were isolated using Todd-Hewitt broth, whereas less than half were isolated from CNA agar plate. Of 58 colonised pregnant women, 12 had strains cultured only from vaginal swabs, while 22 had strains isolated only from the rectal swabs and another 24 had strains isolated simultaneously from both the vaginal and rectal swabs when cultured on a CNA agar plate.

\section{Discussion}

Group B streptococcus is prevalent worldwide and its colonisation rate varies depending on geographical areas and sociodemographic factors. Of the GBS positive cases studied, $52.3 \%$ were women of 20-29 of age. This is an age where sexual activity is increased and GBS is often regarded as sexually transmitted..$^{15}$ The factors that were investigated in this study included reproductive history and HIV status. The high prevalence of HIV infection might be considered to aggravate the burden of GBS disease in South Africa, but maternal HIV infection has not been associated with a high prevalence of GBS colonisation. ${ }^{6}$ No association of HIV status and GBS colonisation was found in the current study. Women with CD4+ lymphocyte counts of $>500$ cells $/ \mathrm{mm}^{3}$ have, however, been reported as an exception with regards to the association. ${ }^{7}$

GBS was previously reported as being associated with preterm delivery, miscarriages, still birth and parity. In various studies, there were no significant differences in colonisation rates noted on the basis of age or parity, though some studies report that increasing age and parity can be associated with lower rates of GBS carriage. ${ }^{16,17}$ Regan et al. ${ }^{18}$ described GBS carriage as being

Table 2: Variables analysed for association with GBS colonization in pregnant women

\begin{tabular}{|c|c|c|c|}
\hline \multirow[t]{2}{*}{ Variables } & GBS colonised $(n=128)$ & GBS non-colonised ( $n=285$ ) & $p$-value \\
\hline & Frequency (\%) & Frequency (\%) & \\
\hline \multicolumn{3}{|l|}{ Gestational age (weeks) } & \multirow[t]{5}{*}{0.12} \\
\hline First trimester & $57(44.5)$ & $56(19.6)$ & \\
\hline Second trimester & $100(78.1)$ & $108(37.9)$ & \\
\hline Third trimester & $10(7.8)$ & $15(5.3)$ & \\
\hline N/A & $35(27.3)$ & $20(7.0)$ & \\
\hline \multicolumn{3}{|l|}{ HIV Status } & \multirow[t]{3}{*}{0.52} \\
\hline Positive & $52(40.6)$ & $104(36.5)$ & \\
\hline Negative & $72(56.3)$ & $170(59.6)$ & \\
\hline \multicolumn{3}{|l|}{ Parity } & \multirow[t]{4}{*}{0.36} \\
\hline 0 & $38(29.7)$ & $66(23.2)$ & \\
\hline $1-2$ & $68(53.1)$ & $160(56.1)$ & \\
\hline$\geq 3$ & $19(14.8)$ & $50(17.5)$ & \\
\hline \multicolumn{3}{|l|}{ Stillbirths } & \multirow[t]{4}{*}{0.24} \\
\hline 0 & $113(88.3)$ & $258(90.5)$ & \\
\hline $1-2$ & $12(9.4)$ & $16(5.6)$ & \\
\hline$\geq 3$ & $0(0.0)$ & $2(0.7)$ & \\
\hline \multicolumn{3}{|l|}{ Miscarriages } & \multirow[t]{4}{*}{0.33} \\
\hline 0 & $93(72.7)$ & $223(78.2)$ & \\
\hline $1-2$ & $29(22.7)$ & $49(17.2)$ & \\
\hline$\geq 3$ & $3(2.3)$ & $4(1.4)$ & \\
\hline
\end{tabular}

Table 3: Comparison between type of media and recovery sites

GBS colonised pregnant women

$128(30.9)$
Type of a media Frequency (\%)

CNA58 (45.3) Todd-Hewitt broth 70 (54.7)
GBS recovery sites Frequency (\%)

Vaginal sites 12 (20.7)

Rectal sites 22 (37.9)

Both sites 24 (41.4) 
more common among older women and women of lower parity. In this study, no association between colonisation and age or parity was found, similar to a study by Valkenburg-van den Berg, et al. ${ }^{19}$ Ethnicity can explain differences in GBS colonisation rates. The GBS colonisation rate among, for instance, black pregnant women in some parts of Africa has been reported to be higher. ${ }^{20}$ Comparisons between different ethnic groups were limited in the current study since all women were of the same ethnic group. Although the importance of infection as a cause of preterm delivery is gaining recognition, little is known about the role of group B streptococcus infection in miscarriages. Conflicting reports have been published to explain the role of GBS infection and miscarriage. ${ }^{21-23}$ There was no association between pregnant women who had a history of miscarriages, stillbirth and group $B$ streptococcus colonisation in the current study.

A comparison of GBS colonisation rate among different studies is often biased due to substantial methodological differences. ${ }^{24}$ The maternal rate of GBS colonisation in this study was $30.9 \%$, which is higher compared to other African countries, such as Mozambique where a colonisation rate of $1.8 \%$ was reported..$^{25}$ The difference in the colonisation rate reported from Mozambique and the present study may be due to the type of samples used for the isolation of GBS. In the Mozambican study they used blood for culturing but in this study only vaginal and rectal swabs were used. Geographic differences can also contribute to the different colonisation rates. Higher rates have been reported previously with $35 \%$ and $54 \%$ in Europe and $47 \%$ in Zimbabwe. ${ }^{26-28}$ The reasons for these high colonisation rates in these studies might also be due to specimen collection and processing techniques used.

Selective broth medium with antibiotics, namely Todd-Hewitt broth with gentamicin and colistin or nalidixic acid, followed by subculture on blood agar was used in this study as recommended by the current Centres for Disease Control and Prevention guidelines for prenatal GBS screening, ${ }^{9,29}$ There is limited data on the validity of this recommended method in low to middle income countries where there are differences in distribution of saprophytic organisms. In the current study, as with Manning et al., ${ }^{14}$ a combination of gentamicin and colistin was used for the recovery of GBS from pregnant South African women instead of combinations of nalidixic acid/colistin and nalidixic acid/ gentamicin. This was done to improve the isolation rate of GBS strains. When direct agar plating was used instead of selective enrichment broth, as many as 50\% of woman who are GBS carriers have false-negative culture results (CDC, 1999). Studies that used selective medium, reported slightly higher rates compared to those that do not. ${ }^{30,31}$ A selective medium containing Todd-Hewitt broth, sheep blood, nalidixic acid and gentamicin was found to enhance the isolation of GBS significantly. ${ }^{32}$ Different areas of specimen collection, may determine the sensitivity in detection of GBS colonisation., ${ }^{43}$ Culture methods should not be neglected for the exclusive use of molecular assays in the detection of GBS.

In conclusion, this study revealed that pregnant women of all ages are at risk of group B streptococcus. The GBS colonisation rate of $30.9 \%$ among pregnant women at Dr. George Mukhari Academic Hospital was comparable to those reported in previous studies. The results confirmed that the recovery of GBS was improved when the use of Todd-Hewitt broth was combined with isolation on CNA plates.
Acknowledgements - We appreciate the assistance of the staff and postgraduate students of the Department of Microbiological Pathology, Sefako Makgatho Health Sciences University, formerly known as the University of Limpopo - MEDUNSA campus.

Conflict of Interest - None of the authors have a commercial or other association that might have posed a conflict of interest concerning the research presented.

Declaration - Sylvester R Moyo received financial support from the National Research Foundation as part of the study "Streptococcus agalactiae in Pregnant Women and their babies at the Dr. George Mukhari Hospital, Pretoria"

\section{References}

1. Shen $X$, Lagergard $T$, Yang $Y$, et al. Preparation and preclinical evaluation of experimental group B streptococcus type III polysaccharide-cholera toxin B subunit conjugate vaccine for intranasal immunization. Vaccine. 2001;19:850-61.

2. Phares CR, Lynfield R, Farley $M M$, et al. Epidemiology of invasive group B streptococcal disease in the United States, 1999-2005. Active Bacterial Core surveillance/Emerging Infections Program Network JAMA. 2008;299(17):2056-65.

3. Sharmila V, Joseph NM, Arun BT, et al. Genital tract group B streptococcal colonization in pregnant women: a South Indian perspective. J Infect Dev Ctries. 2011;5(8):592-5.

4. Yang MJ, Sun PL, Wen KC, et al. Prevalence of maternal group B streptococcus colonization and vertical transmission in low-risk women in a single institute. J Chinese Med Ass. 2012;75:25-8.

5. Zusman AS, Baltimore RS, Fonseca SNS. Prevalence of maternal group $B$ streptococcal colonization and related risk factors in a Brazilian population. The Brazil J Infect Dis. 2006;10(4):242-6.

6. Dangor Z, Lala SG, Cutland CL, et al. Burden of invasive Group B streptococcus disease and early neurological sequelae in South African infants. PLOS ONE. 2015;10(4):e0123014.

7. Cutland CL, Shrag SJ, Thigpen MC, et al. Increased risk for Group $B$ streptococcus sepsis in young infants exposed to HIV, Soweto, South Africa 2004-2008. Research. 2015;21(4):638-45.

8. Busetti M, D'Agaro P, Campello C. Group B streptococcus prevalence in pregnant women from North-Eastern Italy: advantages of a screening strategy based on direct plating plus broth enrichment. J. Clin Path. 2007;60:1140-43.

9. Verani JR, Mcgee L, Schrag SJ. Prevention of Perinatal Group B streptococcal disease revised guidelines from Centres for Disease Control and Prevention MMWR. 2010;59(10):1-32.

10. Gupta C, Briski LE. Comparison of two culture media and three sampling techniques for sensitive and rapid screening of vaginal colonization by Group B streptococcus in pregnant women. J Clin Microbiol. 2004;42:3975-77.

11. Jones N, Oliver K, Jones $Y$, et al. Carriage of Group B streptococcus in pregnant women from Oxford, UK. UK. J Clin Path. 2006;59:363-6.

12. Shet A, Ferrieri P. Neonatal and maternal group B streptococcal infections: a comprehensive review. Indian J Med Res. 2004;120(3):141-50.

13. Lin FY, Philips III JB, Azimi PH, et al. Level of maternal antibody required to protect neonates against early-onset disease caused by Group B streptococcus type ia: a multicenter, seroepidemiology study. J Infect. 2001;184:1022-28.

14. Manning SD, Wood S, Kasha K, et al. Naturally occurring antibodies for the group B streptococcal surface immunogenic protein (Sip) in pregnant women and newborn babies. Vaccine. 2006;24:6905-12.

15. Woldu ZF, Teklehaimanot TK, Waji ST, et al. The prevalence of Group B Streptococus recto-vaginal colonization and antimicrobial susceptibility pattern in pregnant mothers at two hospitals of Addis Ababa, Ethiopia. Reproductive Health. 2014;11:80

16. Anthony BF, Okada DM, Hobel CJ. Epidemiology of Group B streptococcus: longitudinal observations during pregnancy. J. Infect Dis. 1978;137(5):524-30.

17. Yow MD, Leeds LJ, Thompson PK, et al. The natural history of group $B$ streptococcal colonization in the pregnant woman and her offspring I. Colonization studies J Obstet Gynaecol. 1980;137(1):34-8. 
18. Regan JA, Klebanoff MA, Nugent RP. The epidemiology of group B streptococcal colonization in pregnancy Vaginal Infections and prematurity study group. Obstet Gynaecol. 1991;77:604-10.

19. Valkenburg-van den Berg A, Sprij A, Oostvogel P, et al. Prevalence of colonisation with group $B$ Streptococci in pregnant women of a multi-ethnic population in The Netherlands. Eur J Obstet Gynaecol Rep Biol. 2006;124:178-83.

20. Dzowela TK, Igbigbi A. Prevalence of GBS colonization in antenatal women at Queen Elizabeth Central Hospital. Blantyre. Malawi Med J. 2005; 17(3):97-9.

21. McDonald HM, Chambers HM. Intrauterine infection and spontaneous midgestation abortion: is the spectrum of microorganisms similar to that in preterm labor? Infect Dis Obstet Gynecol. 2000;8(5-6):220-7.

22. Daugaard HO, Thomsen AC, Henriques U, et al. Group B streptococci in the lower urogenital tract and late abortions. Am J Obstet Gynecol. 1988;158(1):28-31.

23. El-Kersh T, Al-Nuaim L, Kharfy T, et al. Detection of genital colonization of group B streptococci during late pregnancy. Saudi Med. J. 2002;23(1):447-52.

24. Trijbels-Smeulders MA, Kollée LA, Adriaanse AH, et al. Neonatal group $B$ streptococcal infection:incidence and strategies for prevention in Europe. Pediatr Infect Dis J. 2004;23:172-3.

25. De Steenwinkel Florentien D. O., Tak HU, Muller AE, et al. Low carriage rate of Group B streptococcus in pregnant women in Maputo, Mozambique. Mozambique. Trop Med Int Health. 2008;13:427-9.
26. Bergh K, Stoelhaug A, Loeseth K, et al. Detection of group B streptococci (GBS) in vaginal swabs using real-time PCR with TaqMan probe hybridization. Indian J Med Res. 2004;119(Suppl): 221-3.

27. Bergseng $H$, Bevanger L, Rygg M, Bergh K. Real-time PCR targeting the sip gene for detection of Group B streptococcus colonization in pregnant women at delivery. J Med Microbiol. 2007;56:223-8.

28. Mavenyengwa RT, Maeland JA, Moyo SR. Putative novel surfaceexposed streptococcus agalactiae protein frequently expressed by the Group B streptococcus from Zimbabwe. Clin Vaccine Immunol. 2009;16:1302-8.

29. Kwatra G, Madhi SA, Cutland CL, Buchmann EJ, Adrian PV. Evaluation of trans-vag broth, colistin-nalidixic agar, and chromagar strepb for detection of Group B streptococcus in vaginal and rectal swabs from pregnant women in South Africa. J. Clin. Microbiol. 2013;51 (8): 2515-9.

30. Valkenburg-van den Berg A, Sprij A, Oostvogel $P$, et al. Prevalence of colonisation with group $B$ streptococci in pregnant women of a multi-ethnic population in The Netherlands. Eur J Obstet Gynaecol Rep Biol. 2006;124:178-83.

31. Madzivhandila $M$, Adrian PV, Cutland CL, et al. Serotype distribution and invasive potential of Group B Streptococcus isolates causing disease in infants and colonizing maternal-newborn dyads. PLoS ONE. 2011;6(3):e17861.

32. Baker CJ, Clark DJ, Barrett FF. Selective broth medium for isolation of group B streptococci. Applied microbiology. 1973;26(6): 884-5.

33. Stoll BJ, Schuchat A. Maternal carriage of group B streptococci in developing countries. Paed Infect Dis J. 1998;17:499-503.

Received: 10-03-2015 Accepted: 08-10-2015 